University of Wollongong

Research Online

Faculty of Social Sciences - Papers (Archive) Faculty of Arts, Social Sciences \& Humanities

2001

Perceptual grouping in two visually reliant species: Humans (Homo sapiens) and Australian sea lions (Neophoca cinerea)

Darren Burke

University of Wollongong

Paul Everingham

University of Wollongong

Tracey Rogers

University of Sydney

Melinda Hinton

University of Wollongong

Sophie Hall-Aspland

University of Sydney

Follow this and additional works at: https://ro.uow.edu.au/sspapers

Part of the Education Commons, and the Social and Behavioral Sciences Commons

Research Online is the open access institutional repository for the University of Wollongong. For further information contact the UOW Library: research-pubs@uow.edu.au 


\title{
Perceptual grouping in two visually reliant species: Humans (Homo sapiens) and Australian sea lions (Neophoca cinerea)
}

\author{
Abstract \\ Kurylo, van Nest, and Knepper (1997 Journal of Comparative Psychology 111126 - 134) have recently \\ shown that hooded rats are able to judge the global orientation of an array of elements if orientation is \\ signalled by the perceptual-grouping principle of proximity, but not if it is signalled by element alignment. \\ Using a procedure designed to overcome some potential problems with the experiment of Kurylo et al, we \\ found the same distinction in the perceptual processing of Australian sea lions. The sea lions were able to \\ judge the orientation of arrays containing strong proximity and similarity information, but performed at \\ chance levels judging arrays in which element alignment signalled global orientation. Human subjects \\ were able to judge all three pattern types quickly and accurately. This is strong evidence of a qualitative \\ distinction in the way in which perceptual grouping operates in humans and the non-human species \\ tested. Whether this distinction is a consequence of evolutionary or experiential factors is a question for \\ future research, but the mere fact of a qualitative difference holds important implications for our \\ understanding of perceptual grouping.
}

\section{Keywords}

neophoca, lions, sea, australian, sapiens, cinerea, homo, perceptual, humans, species, reliant, visually, two, grouping

\section{Disciplines}

Education | Social and Behavioral Sciences

\section{Publication Details}

Burke, D., Everingham, P., Rogers, T., Hinton, M. \& Hall-Aspland, S. (2001). Perceptual grouping in two visually reliant species: Humans (Homo sapiens) and Australian sea lions (Neophoca cinerea).

Perception, 30 (9), 1093-1106. 


\title{
Perceptual grouping in two visually reliant species: Humans (Homo sapiens) and Australian sea lions (Neophoca cinerea)
}

Darren Burke, Paul Everingham, Tracey Rogers $\uparrow$, Melinda Hinton, Sophie Hall-Aspland $\uparrow$ Department of Psychology, University of Wollongong, Wollongong, NSW 2522, Australia; Australian Marine Mammal Research Centre, Taronga Zoo/Sydney University, Mosman, NSW 2088, Australia Received 24 November 2000, in revised form 20 May 2001

\begin{abstract}
Kurylo, van Nest, and Knepper (1997 Journal of Comparative Psychology 111 126-134) have recently shown that hooded rats are able to judge the global orientation of an array of elements if orientation is signalled by the perceptual-grouping principle of proximity, but not if it is signalled by element alignment. Using a procedure designed to overcome some potential problems with the experiment of Kurylo et al, we found the same distinction in the perceptual processing of Australian sea lions. The sea lions were able to judge the orientation of arrays containing strong proximity and similarity information, but performed at chance levels judging arrays in which element alignment signalled global orientation. Human subjects were able to judge all three pattern types quickly and accurately. This is strong evidence of a qualitative distinction in the way in which perceptual grouping operates in humans and the non-human species tested. Whether this distinction is a consequence of evolutionary or experiential factors is a question for future research, but the mere fact of a qualitative difference holds important implications for our understanding of perceptual grouping.
\end{abstract}

\section{Introduction}

The ability to perceptually 'group' together different parts of the same object, and to be able to distinguish this 'grouping' from other objects, is fundamental to any reasonably sophisticated visual system. It is clear, from the very fact that visually reliant animals successfully navigate through the world, that many species are capable of some form of visual perceptual grouping, but it is not clear from such observations what information they are using to accomplish this. As the Gestalt psychologists pointed out (Koffka 1935, for example), there are many sources of grouping information available. Objects are typically composed of parts that are close together (proximity), similar in colour and shape (similarity), follow smooth contours (good continuation), and they usually move in unison, at least on a large scale (common fate). Conversely, elements which differ in any of these dimensions are usually parts of different objects. Except in the case of camouflage (in which conflicting grouping information is provided), grouping cues almost always provide congruent information, so it is possible that not all visually reliant animals use all such sources of grouping information. (1) Since scene segmentation is a fundamental aspect of vision, it seems likely that information about which animals use which grouping cues will help us to understand the evolution and ecology of visual mechanisms, as well as being a means by which we may be able to tease apart mechanisms which may appear unitary in human perception.

Kurylo et al (1997) recently showed that rats (Rattus norvegicus) are sensitive to global orientation defined by the perceptual-grouping principle of proximity, but not to orientation defined by element alignment (a version of good continuation). The rats

(1) Since camouflage generally does involve providing conflicting grouping information, and since grouping information is usually redundant in other situations, animals which prey on camouflaged species may face selection pressure to rely less strongly on the cue that is providing the conflicting information. This may render them better able to detect the prey species without sacrificing their general ability to perceptually group the visual world. 
were trained to discriminate vertical from horizontal (using arrays of three horizontal and three vertical lines), and were then tested for the degree to which this training transferred to stimuli in which orientation was defined exclusively by the arrangement of small, square elements presented in an array. When we view such arrays, we see them as vertical if the elements are aligned vertically but not horizontally (this is alignmentsee figure 1a) or if they are closer together vertically than they are horizontally (this is proximity - see figure 1b). The surprising conclusion of the Kurylo et al experiment is that rats also see the array defined by proximity as vertical, but are unable to use element alignment as a cue to orientation.
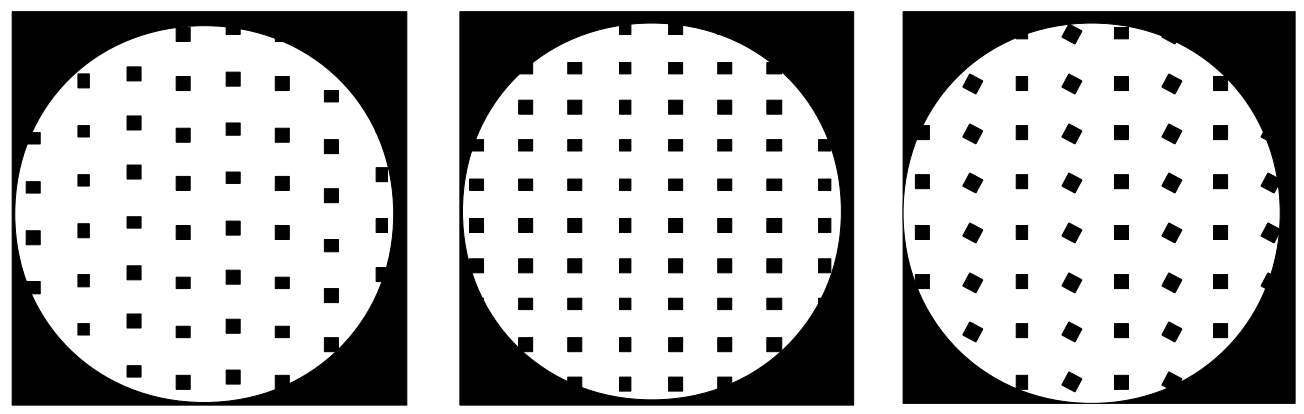

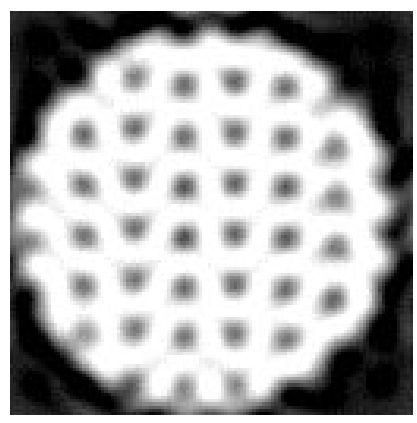

(a)

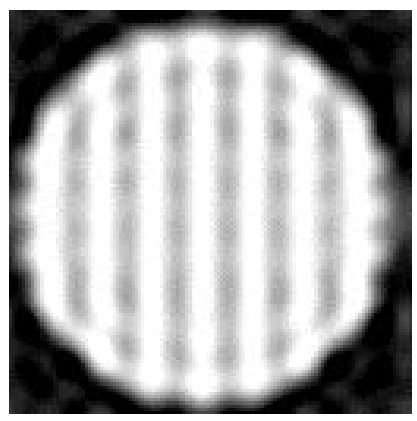

(b)

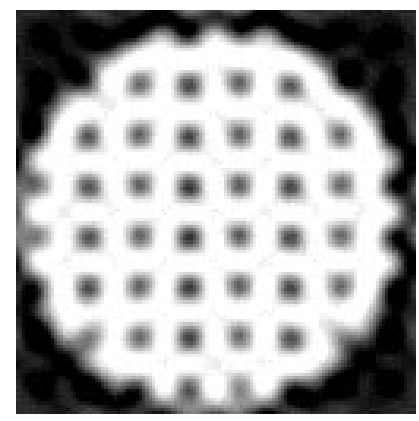

(c)

Figure 1. Vertical versions of the three grouping principles tested in the current experiments, and low-pass spatial-frequency-filtered images of them. (a) Vertical by alignment; (b) vertical by proximity; (c) vertical by similarity (achieved by rotating elements). The low-pass filtering of the stimuli produces a clear vertical signal only in the proximity condition (see text for details).

This suggests that there may be a fundamental difference in the way in which perceptual grouping operates in the human and rat visual systems, even within grouping mechanisms that are based on the spatial arrangement of the elements. Of course, such a conclusion could only be convincingly drawn if other differences between the subjects used in these experiments were ruled out as possible causes of the perceptual difference. As discussed below, differences in visual acuity between rats and humans can explain the results of Kurylo et al, but there are also other differences which are probably important. For example, the visual environments of the human subjects and the laboratory rats were almost certainly very different (laboratory rats live in much less visually rich environments), and this may make (or have made, developmentally) an important difference to the kinds of grouping cues they are sensitive to.

A qualitative difference like that identified by Kurylo et al is the strongest possible evidence that there is an important disparity between the perceptual processes of two species, and is, therefore, worthy of thorough investigation. If such a distinction is valid, it raises a range of questions about how evolution may have produced the difference, 
what mechanisms underlie it, and what implications it holds for other possible differences between the species. It is because of the potential importance of such qualitative differences that we decided to compare perceptual grouping in humans and in another non-human, but visually reliant, species. Our primary aims were to extend the species used in such comparisons (particularly to another that depends on vision) while attempting to rule out some potentially less interesting explanations of the Kurylo et al findings. If we are to use Kurylo et al's results to guide investigations of comparative perception, then it is important to be sure that the difference they observed is actually caused by a difference in some higher-order perceptual-grouping mechanism and is not a relatively uninteresting consequence of the way in which the animals were trained, or of the particular stimuli used in the experiment. Although they carefully manipulated the strength of the grouping cues, ruling out some possible problems, there remains a somewhat less obvious competing explanation, which may compromise the validity of their results.

One explanation of the results of Kurylo et al which they do not fully explore is the possibility that the rats had rather poor acuity, and that they therefore saw the test patterns much less sharply, than do human observers. The usual way to compare acuity across species is in terms of contrast sensitivity functions - plots of the lowest contrast at which arrays of (usually) vertical gratings of a range of spatial frequencies can be just detected. Humans are most sensitive to gratings with a spatial frequency of between 1 and 5 cycles $\mathrm{deg}^{-1}$, but at reasonable contrasts can detect gratings over a much wider range (between about 0.1 and 40 cycles $\mathrm{deg}^{-1}$ ). As Kurylo et al note, hooded rats, like those used in their study, cannot see gratings above about $1.5 \mathrm{cycles} \mathrm{deg}^{-1}$, no matter how high their contrast, and are more sensitive to spatial frequencies below those that humans can detect (Birch and Jacobs 1979; Legg 1984). This means that, if we are to estimate the information available to a rat in a visual scene, we need to remove the high-spatial-frequency information from it by using a low-pass spatial-frequency filter of the image. Such a technique will not allow us to see the scene as the rat does, since, for one thing, the rat is more sensitive to low-frequency information than are we, but it will at least remove from the image the information that the rat does not have available to it.

As is shown in figure 1, filtering out the high-spatial-frequency information from vertical stimuli defined only by proximity produces clear vertical lines, ${ }^{(2)}$ but removing the same high-frequency information from patterns in which verticality is defined by alignment produces a much weaker vertical signal. The difference between the lowpass filters of the patterns in figure 1 is, in fact, much less dramatic than would be a similar filtering of the patterns used by Kurylo et al, since our alignment pattern maintains the vertical spacing between the elements, whereas they varied the vertical spacing randomly in the alignment condition. This had the effect of creating some large vertical gaps between elements in the alignment condition, which would reduce the strength of the low-frequency vertical signal. A simple blurring of the example patterns they provided (their page 129) makes this point obvious.

It seems at least possible from these considerations that the rats accurately judged the orientation of the proximity patterns, but not of the alignment patterns, because, to them, only the former strongly resembled the training stimuli. Indirect support for the possibility that this underlies the perceptual discrepancy discovered by Kurylo et al is provided by another recent experiment using very similar stimuli with human observers (Kurylo 1997). In this experiment Kurylo discovered that the time needed to judge the orientation of a pattern was longer for patterns in which orientation was

(2) Ginsburg (1986) has argued, in fact, that information in low-spatial-frequency channels is sufficient to explain grouping by proximity in humans (along with a host of other perceptual effects). Only the proximity patterns can be discriminated in this way in the current experiments. 
defined by alignment than when it was defined by proximity. Kurylo suggests that this difference could be due to judgments based on alignment being more computationally intensive than those based on proximity, but, again, differences in the usefulness of lowfrequency information may be sufficient to explain the difference. If the proximity (but not the alignment) stimuli can be discriminated on the basis of low-spatial-frequency information, then we might expect them to be judged more quickly because information in the magnocellular pathway is sufficient to discriminate them, and the magnocellular pathway responds more quickly than the parvocellular pathway (Livingstone and Hubel 1987, for example).

Similarly, if a rat has available to it only low-frequency information, then it is not surprising to discover that it is much better able to judge the orientation of the patterns that contain strong, low-frequency orientation information than of those that do not. In fact, we might expect the discrepancy between grouping on the basis of proximity and alignment discovered by Kurylo et al (1997) even if rats had no mechanism at all for grouping together discrete elements, or if they had been trained in such a way that they did not engage such a mechanism when exposed to the test patterns. It seems to us possible that training subjects with just one exemplar of vertical and one of horizontal as Kurylo et al (1997) did - may be insufficient to ensure that they are using the 'concept' of verticality and/or horizontality to perform the discrimination. If they are not, then they will be able to successfully discriminate test stimuli which are broadly similar to the training exemplars (like the proximity patterns), but they may find discriminations between less obviously similar stimuli (like the alignment patterns) more difficult. Perhaps if the training had encouraged the rats to examine the patterns for any evidence of global orientation, they may have been able to perform above chance on the alignment test patterns. We indirectly explore this possibility in the current experiments.

As was outlined earlier, the experiments reported here are designed to examine in more detail the interesting possibility that there are species differences in the ability to use perceptual-grouping principles to segregate the visual world. We decided to examine whether the same distinction is evident in the way in which two highly visually reliant species use perceptual-grouping information. The non-human species (Australian sea lions) were chosen primarily because they are representatives of highly visually reliant mammals, and because they almost certainly have quite good in-air visual acuity (see below). Of course, this species is also amphibious, and it has also been selected as an experimental subject so that in later studies the effect that this may have had on the functioning of their visual system could be examined, but this aspect is not relevant to the rationale of the current study. We are not predicting that adaptation to seeing underwater will have made a difference to the way in which these animals use grouping information.

We took a number of steps in an attempt to overcome the potential problems with the study of Kurylo et al (1997) discussed above:

(i) We selected a non-human species which depends strongly on vision. Australian sea lions are active hunters which locate and capture underwater prey using vision (Bonner 1994). There are no data available for this species, but Steller sea lions (Eumetopias jubatus; Schusterman 1981) and California sea lions (Zalophus californianus: Schusterman and Balliet 1971) have similar in-air acuity, which is comparable to that of cats. Since these species are the closest living relatives of Australian sea lions there is no reason to expect our subjects' acuity to be substantially different from theirs.

(ii) We trained the sea lions with a range of horizontal and vertical exemplars. This was designed to encourage them to use the 'concept' of vertical and/or horizontal, rather than learning particular exemplars [Kurylo et al (1997) used one of each] in order to give the animals the best chance of demonstrating sensitivity to the grouping principles they were presented with-in other words, we wanted them to respond on the basis of the stimulus containing any evidence of verticality and/or horizontality. 
(iii) We tried to partially control for the effects of differential acuity by including a grouping by similarity condition, in which the low-pass orientation information is no more useful than it is in the alignment condition. If the animals can group using such a similarity cue, but are unable to use the alignment information, then this cannot be a simple consequence of poor acuity. An ideal control for this possibility would have been to construct grouping patterns from elements which contain information at only a single spatial frequency (or in a very narrow band), so that a low-pass filter will remove all of the orientation information, and so be of no use for performing the task. We did not do this in this study for two reasons. First, producing such patterns for use in the home enclosure of the animals was logistically difficult, since we could not be sure that the stimuli produced signals that were restricted to a single band of frequencies using the computers and monitors we had available to us. Second, there is no precise information about the acuity of the species we used, and the two sea lion subjects may have differed somewhat in acuity, so to implement this kind of control we would have had to measure the acuity of each of the subjects prior to producing the patterns.

\section{Experiment 1: Humans (Homo sapiens)}

Experiment 1 was designed as a baseline against which we could compare the performance of the non-human subjects. The main function of this experiment was to demonstrate that the stimulus parameters we chose led to reliable percepts of horizontal and vertical, and that our manipulation of cue strength succeeded in producing patterns which did actually differ in this way.

Alignment and proximity were used in this experiment because they were the cues used by Kurylo et al (1997). The results of Kurylo et al are particularly surprising because both of these grouping cues rely on the spatial arrangement of the elements to convey orientation information. It would perhaps have been less surprising, and less important, if they had discovered that rats were sensitive to grouping information provided by, for example, shape or luminance similarity, but not by spatial arrangements. In such a case the grouping information would be carried in different visual modalities, and differential sensitivities to luminance and spatial position between the species could easily account for the results.

As was discussed earlier, the current experiment contains a condition in which overall orientation is defined by element similarity. Element similarity was manipulated by changing the orientation of the square elements (see figure 1c). This has two advantages in the current context. First, it is a similarity cue which is carried by highspatial-frequency information, so a low-pass filter of the image provides no global orientation cue, as discussed previously. Second, element orientation is, in a sense, a grouping cue defined by the spatial arrangement of the elements, in common with proximity and alignment. It is true that the grouping cue is not related to spatial location in this case, but the elements are all similar, as they are in the alignment and proximity cases, which they would not be in other, more typical, manipulations of similarity.

\subsection{Methods}

2.1.1 Subjects. Thirty undergraduate psychology students from the University of Wollongong served as subjects in the experiment for nominal course credit. There were twelve males and eighteen females. All subjects had normal or corrected-to-normal vision.

2.1.2 Apparatus. The experiment was run in a laboratory on a PowerMac 6200. ClarisWorks drawing was used to generate the pictures used. RSVP (Williams and Tarr, no date) was used to present the stimuli to the subjects and record their reaction times and errors. 
2.1.3 Stimuli. Subjects were presented with the grouping stimuli either as pairs (one horizontal by grouping cue, the other vertical) or individually. These will be referred to as the dual-picture phase and the single-picture phase, respectively. In the dual-picture phase subjects were required to judge which of the patterns was vertical (on the left or the right of the screen), and in the single-picture phase they were required to judge whether the pattern was vertical or horizontal. Within each of these presentation phases, there were six different stimulus types. There were two levels of each of the three grouping cues [alignment, similarity (rotation), and proximity]; one was a 'strong' version of the cue and one a 'weak' version. Pilot testing was used to determine roughly equivalent cue strengths across the different kinds of cues. We selected values that produced, in the 'strong' case, a fairly obvious percept of global orientation, and another 'weak' which produced a percept of global orientation that was less obvious, and generally took some time to detect. The precise parameters used to produce the stimuli are described below.

In the dual-picture phase of the experiment, the elements were $5 \mathrm{~mm} \times 5 \mathrm{~mm}$ squares. Before imposing a grouping cue on the elements, they were equally spaced $8 \mathrm{~mm}$ apart (perturbations to this arrangement produced the grouping information, as described below). In the dual-picture phase the outside edges of the two stimuli were occluded by a black mask with circular apertures $65 \mathrm{~mm}$ in diameter. The centres of the stimuli were separated horizontally by $118 \mathrm{~mm}$. The two arrays contained elements that were arranged so that one array had the grouping cue signalling vertical and the other signalled horizontal. Grouping cue type and strength were always paired, so that, for example, a weak proximity cue to vertical was always paired with a weak proximity cue to horizontal.

In the single-picture phase there was no occluding mask (the stimulus was square), and the size of the stimulus was $150 \%$ larger than the corresponding stimulus in the dual-picture phase. This was the same size and shape as the stimuli presented to the sea lions, except that they were presented with the stimuli as pairs (one on each of two monitors). We could not easily measure reaction time for the humans with stimuli presented on two monitors, mimicking the conditions for the sea lions, so we ran these two versions of the task with our human subjects in order to investigate whether there were effects which may have been specific to the size and shape of the stimuli used, or to presenting the stimuli as pairs.

For each of the stimulus arrangements used, measurements are reported for the dual-picture phase stimuli. The single-picture phase versions are simply $150 \%$ larger in all measurements (except rotation). The head position of the subjects was not controlled (since it could not be for the sea lions), but viewing distances were typically in the range of $50-60 \mathrm{~cm}$, at which $10 \mathrm{~mm}$ is approximately $1 \mathrm{deg}$ of visual angle.

Proximity metrics. In the strong vertical condition the vertical spacing of the elements remained at $8 \mathrm{~mm}$ and the horizontal spacing was increased to $13.5 \mathrm{~mm}$. The opposite arrangement was used for the strong horizontal condition. In the weak vertical condition the vertical spacing was $8 \mathrm{~mm}$ and the horizontal spacing was $11.25 \mathrm{~mm}$. See figure 1 for an example.

Alignment metrics. In order to create a stimulus which was vertical by alignment information, all of the elements in columns 1 and 5 were moved upwards (every element in the column was moved the same amount), and every element in columns 3 and 7 were moved downwards. Elements in columns 2, 4, 6, and 8 were not moved. This resulted in a stimulus in which the elements were aligned vertically but not horizontally. See figure 1 for an example. In the strong version of the cue the elements were moved $4.5 \mathrm{~mm}$ and in the weak version they were moved $1 \mathrm{~mm}$. In the single-picture phase, this manipulation resulted in a stimulus which had a smooth outline in one dimension (in the orientation being cued) and an irregular outline in the non-cued orientation. 
To prevent subjects from using this cue to make the orientation judgment (particularly the sea lion subjects who had stimuli like those in the single-picture phase), several elements were randomly removed from the edges with smooth outlines, to make them irregular.

Rotation metrics. The elements in these stimuli were equally spaced $(8 \mathrm{~mm})$, but each element in every second column was rotated $25^{\circ}$ to the right for the strong vertical grouping cue, or $3^{\circ}$ to the right for the weak vertical grouping cue. See figure 1 for an example.

\subsection{Procedure}

Each subject was tested individually. All subjects were exposed to all conditions. General instructions were given to each subject verbally and written instructions were given on the screen prior to commencement of trials.

Subjects were asked to attend to a small, centralised fixation cross before each trial. The trials were self-paced and subjects proceeded by pressing the space bar. The stimuli then appeared and persevered until subjects made their choice or failed to make a choice within $12 \mathrm{~s}$. In the dual-picture phase subjects were required to indicate the side of the screen that contained the vertical configuration. If they perceived the circle on the right side of the screen as containing the vertical pattern, they pressed the ' $p$ ' key and if it was on the left they pressed the 'q' key. As mentioned previously, the stimulus types were presented as pairs with vertical and horizontal patterns of the same type appearing together. The side on which the vertical pattern appeared was counterbalanced for each stimulus type.

In the single-picture phase subjects had to decide whether the pattern presented was vertical or horizontal. The ' $\mathrm{v}$ ' key indicated that the picture was vertical and the ' $h$ ' key indicated a horizontal pattern. Subjects were asked to make their responses as quickly and accurately as possible.

The dual-picture phase consisted of a block of 24 trials. There were two presentations of each stimulus pairing in which the stimulus on the left was vertical and two in which the stimulus on the right was vertical. The order of presentation was randomised for each subject. The single-picture phase consisted of a block of 24 trials (four presentations of each stimulus, twice vertically and twice horizontally), also in random order. The order of the two presentation phases (single picture and dual picture) was counterbalanced across subjects.

\subsection{Results}

The measure of each subject's reaction time was obtained by calculating the mean reaction time for all correct responses in a given stimulus condition for that subject. These mean reaction times were analysed with two 2 (cue strength) $\times 3$ (grouping principle) repeated-measures factorial ANOVAs, one for the dual-picture phase and one for the single-picture phase. Errors were also recorded and total errors for each subject in a given condition (out of a possible 4 ) were analysed in two similar $2 \times 3$ repeatedmeasures ANOVAs.

As can be seen by comparing figures $2 \mathrm{a}$ and $2 \mathrm{c}$, and $2 \mathrm{~b}$ and $2 \mathrm{~d}$, the pattern of results was broadly similar in the dual-picture phase and in the single-picture phase. In the analysis of the reaction time data from the single-picture phase there was a significant main effect of cue strength $\left(F_{1,29}=15.47, p=0.0005\right)$, but no main effect of grouping cue $\left(F_{2,58}=2.38, p=0.10\right)$, and no interaction $\left(F_{2,58}=1.63, p=0.21\right)$. The error data from the single-picture phase revealed a significant main effect of cue strength $\left(F_{1,29}=19.00, p=0.0001\right)$, and a main effect of grouping cue $\left(F_{2,58}=10.45\right.$, $p=0.0001)$, but no interaction $\left(F_{2,58}=1.04, p=0.37\right)$. So, in the single-picture phase the manipulation of difficulty appears to have been successful. The strong cues are detected more quickly and more accurately than the weak cues. Despite attempts to equalise the relative difficulty of the cues within each of the strengths, some differences between them did emerge. 


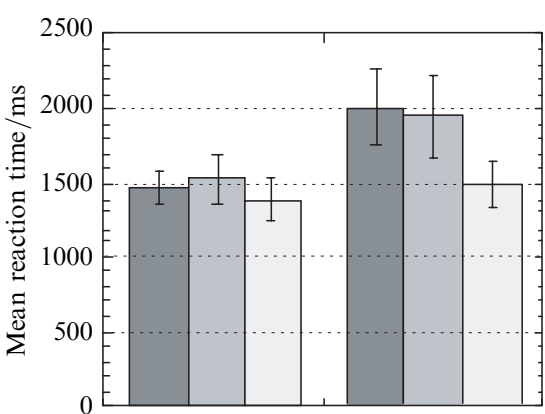

(a)

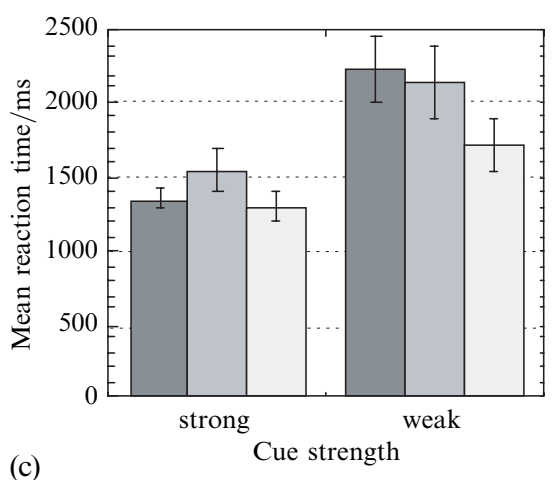

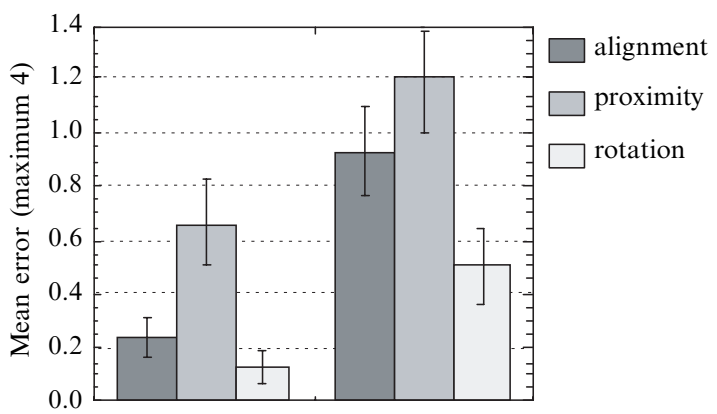

(b)

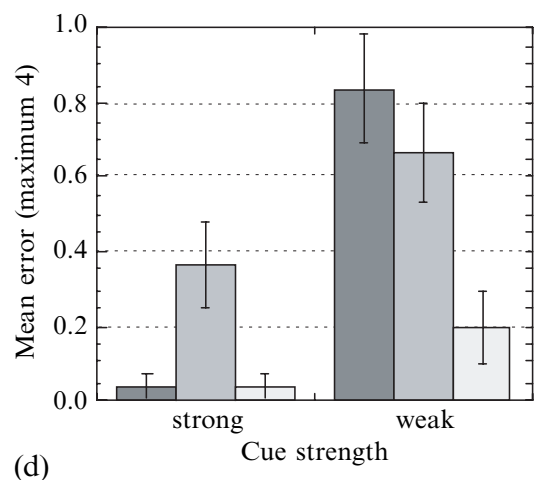

Figure 2. Results from human subjects responding to the three grouping cues in experiment 1 . Error bars represent \pm 1 standard error of the mean. (a) Mean reaction time in the singlepicture phase; (b) mean errors (out of a maximum of 4) in the single-picture phase; (c) mean reaction times in the dual-picture phase; (d) mean errors (out of a maximum of 4 ) in the dualpicture phase.

The results are very similar for the dual-picture phase. The reaction time data are almost identical to those collected in the single-picture phase, with a significant main effect of cue strength $\left(F_{1,29}=25.48, p=0.0001\right)$, as well as a significant main effect of grouping cue $\left(F_{2,58}=3.68, p=0.031\right)$, and a significant interaction $\left(F_{2,58}=3.30\right.$, $p=0.044)$ at the 0.05 level. The error data show a similar pattern, with a significant main effect of cue strength $\left(F_{1,29}=22.56, p=0.0001\right)$, a significant main effect of grouping cue $\left(F_{2,58}=8.02, p=0.0008\right)$, and a significant interaction $\left(F_{2,58}=6.33\right.$, $p=0.033)$. As with the single-picture phase, the largest effect was of cue strength, but there were also some differences between the grouping cues.

\subsection{Discussion}

The fundamental aim of this experiment was to ensure that the stimulus parameters we selected to signal global orientation produced reliable percepts of horizontal and vertical, and that the strong versions of the cues were easier to detect than the weak versions. These aims were achieved. It would, perhaps, have been ideal to have stimulus parameters which produced only a significant main effect of cue strength, but this proved difficult to achieve. The parameters we used were selected on the basis of pilot observations by the authors and naïve observers in which we attempted to equalise the strength of the grouping cues, but systematic differences nevertheless emerged. In the context of assessing whether non-humans are sensitive to alignment cues, it is important that the biggest differences between the strong and the weak versions are for this cue. Given this fact, if the sea lions do not show sensitivity to alignment, but do show sensitivity to the other two cues, it would be very difficult to explain this in terms of the interactions uncovered in the results of this experiment. 
In terms of comparing the results of the sea lions and the humans, it is also important that the results from the dual-picture phase and the single-picture phase are so similar. This is despite the fact that in the single-picture phase the stimuli were $150 \%$ larger, they were not surrounded by circular masks, and they had to be judged in isolation. This suggests that the prime determinant of performance in these tasks is the strength and nature of the grouping cue in question, rather than the way in which it is presented, or the exact nature of the decision to be made. This is obviously important in the current study because there are necessarily differences between the way in which the sea lions and the humans are exposed to the stimuli, and may well be differences in the nature of the decision they make. Although the sea lions will be exposed to both a vertical and a horizontal pattern on each trial, as in the dual-picture phase for the humans, they may not base their responses on a comparison between the stimuli. It is encouraging, therefore, to discover that the responses of the human subject were not substantially altered by the manner in which the stimuli were presented (paired or in isolation).

\section{Experiment 2: Australian sea lions (Neophoca cinerea)}

As explained previously, the primary aim of the current research is to further explore the finding of Kurylo et al (1997) that rats are sensitive to orientation information provided by the perceptual-grouping principle of proximity, but are not sensitive to an orientation signal carried by alignment information. The current study is an attempt to test whether the same distinction is true for a much more visually reliant species, while attempting to rule out ways by which a behavioural difference may emerge in the absence of a genuine qualitative dichotomy in their use of grouping information.

\subsection{Method}

3.1.1 Subjects. The subjects were two mature male Australian sea lions (Neophoca cinerea), Kotick and Orson, who were housed at Taronga Zoo in Sydney. They have been in captivity, and have been trained to perform simple husbandry behaviours since they were pups. These behaviours involve them responding to verbal and visual signals to enable them to be moved between pools, inspected by veterinarians, etc.

3.1.2 Stimuli. The stimuli used in the test phase of this experiment were the same as those used in the single-picture phase of experiment 1 , except that the sea lions were simultaneously presented with a horizontal and a vertical version of the cue (as in the dual-picture phase in experiment 1) on two separate monitors. In addition to the two strengths of each of the three grouping cues, the sea lions were also tested with a novel exemplar of the kind of stimuli used in the training phase. This served as a baseline measure of their performance on new patterns which contained real vertical and horizontal lines (which did not require sensitivity to any form of grouping information).

In the training phase the sea lions were taught the vertical/horizontal discrimination using 20 different exemplars of horizontal and vertical. The vertical exemplars used are shown in figure 3 . The training stimuli were designed to be sufficiently complex that they would encourage the sea lions to examine them for evidence of verticality or horizontality. This was an attempt to give the sea lions the best chance of demonstrating their possible percepts of horizontal and vertical in the test patterns, since they were accustomed to responding to stimuli which differed in many ways except that one always contained a vertical line (or lines) and the other a horizontal line (or lines).

3.1.3 Apparatus. The experiment was conducted in a hallway which was part of the indoor facility adjacent to the sea lions' home pools at Taronga Zoo. The apparatus consisted of two IBM-compatible 486 PCs mounted behind a board with two screensize (14-inch) Plexiglass windows installed. The stimuli were presented with PCXshow ${ }^{\circledR}$. 

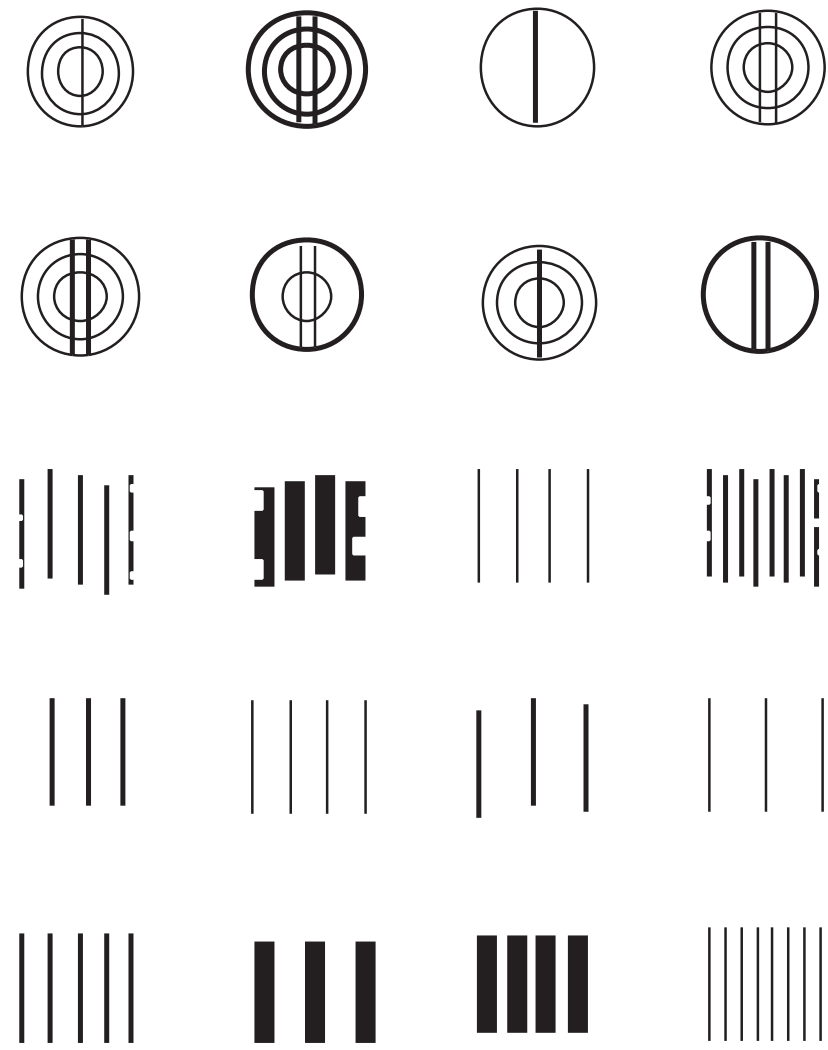

Figure 3. Vertical exemplars of the training stimuli used with the sea lions.

A divider projected $500 \mathrm{~mm}$ perpendicularly between the two windows. This was designed to prevent the sea lions from examining the stimuli from an acute oblique angle. Because of foreshortening due to perspective, this introduces spurious proximity information to stimuli which are actually equally spaced if viewed from in front. The divider forced them to make their judgments from at least $500 \mathrm{~mm}$ from the stimuli.

\subsection{Procedure}

The basic procedure was the same across the three phases of the experiment (pre-training, training, and testing). The sea lion was held by a zoo trainer at a station some $3 \mathrm{~m}$ from the apparatus. A verbal cue was then given to orient the animals head towards the apparatus ('look out'). The verbal send cue ('target') was then given and the animal made its way to the apparatus. These behaviours were trained before the sea lions were exposed to the training stimuli. Once the training stimuli had been introduced, the sea lions made their choice by positioning their nose on a picture. The trainer then either gave a verbal secondary reinforcer ('good') and a fish reward or the verbal cue ('no') according to the reinforcement contingency (vertical S+/horizontal $\mathrm{S}-$ ). This completed the trial, after which the animal was called back by name to the stationing point, and the procedure repeated.

3.2.1 The pre-training phase. Before moving all stimuli to computer screens, the sea lions were trained with an apparatus identical to that already described except that the training stimuli were on laminated sheets of A4 paper and held behind the Plexiglass by Velcro tabs. Since there was some chance that the refresh rate of the computer monitors would create difficulties for the sea lions, we wanted to ensure that early training was with stimuli we were sure they could clearly resolve. 
Which 12 of the 20 training stimuli were to be used in a training session was decided randomly. Whether the $\mathrm{S}+$ (vertical) was on the left or the right side for a given trial was determined by following Gellerman's (1933) schedule. Training continued in this phase until both sea lions reached a criterion of $83 \%$ correct $(10 / 12)$ for four consecutive sessions.

3.2.2 The training phase. The training phase involved continuing to train the sea lions to discriminate between vertical $(\mathrm{S}+)$ and horizontal $(\mathrm{S}-)$, with the stimuli shown in figure 3, but with the stimuli presented on computer monitors. A training session consisted of the presentation of three different preprogrammed blocks of twelve trials. There were usually two, but occasionally three training sessions per day.

Because the stimuli were presented on two different computers, but needed to be matched (a horizontal of one sort on the left monitor had to coincide with a vertical of the same sort on the other monitor), it was not possible to use a new random order of stimuli in each session. Instead, fifteen different 12-stimulus sequences (and their opposites, so that horizontal and vertical versions could be matched) were created, by randomly selecting 12 of the 20 training stimuli, and then randomly selecting whether the stimulus would be horizontal or vertical. Before each training session, the three sequences to be used were selected randomly. Each 12-trial sequence on a given computer consisted of $6 \mathrm{~S}+$ and $6 \mathrm{~S}-$ stimuli, so that horizontal and vertical were counterbalanced for side of appearance.

3.2.3 The test phase. In the test phase, the 7 test stimuli were interspersed randomly into 7 new sets of trials (and their opposites). Each of the new sets of trials contained 4 test stimuli (and therefore 8 training stimuli). Test stimuli were reinforced nondifferentially (the sea lions received a 'good' and a fish regardless of which stimulus they chose). This was to ensure that the sea lions could not learn which of the test stimuli were correct. We were interested in their perception of the test patterns, not in their ability to learn to respond to the vertical pattern on the basis of differential reinforcement. This aim could also have been met by not rewarding the sea lions, irrespective of the test stimulus chosen (testing during extinction, effectively), but, since male sea lions are potentially dangerous when they are frustrated, we decided not to do this.

Results on the test stimuli were included in the analysis only if performance on the non-test stimuli in that session was at $75 \%$ or above (6 out of 8 ). This was to ensure that we included data only from sessions in which the sea lions were properly motivated and attentive. Testing ended when each animal had been exposed to each of the test stimuli 20 times (after substandard sessions were excluded).

\subsection{Results}

Figure 4 shows the performance of the sea lions in the pre-training and training phases of the experiment. Early deviations from chance performance were usually a consequence of side biases. Both sea lions had met the criterion for moving from the pre-training to the training phase after thirty-seven sessions, although Kotick demonstrated consistent above-chance performance before this. Transferring to the computer displays of the training stimuli led to a brief drop in performance, but both animals rapidly recovered to be performing above chance by the third or fourth session of the training phase. After fifteen sessions in the training phase, the subjects were moved to the test phase.

Figure 5 shows performance on the test stimuli. Baseline stimuli are previously unseen stimuli which contain real horizontal or vertical lines (like the training stimuli), which were nondifferentially reinforced, like the other test stimuli. They are included for comparison with the grouping stimuli. 15 correct responses out of 20 exposures represents above-chance performance on a $\chi^{2}$ or a binomial test. By this criterion, both sea lions performed above chance on the strong versions of the proximity and 


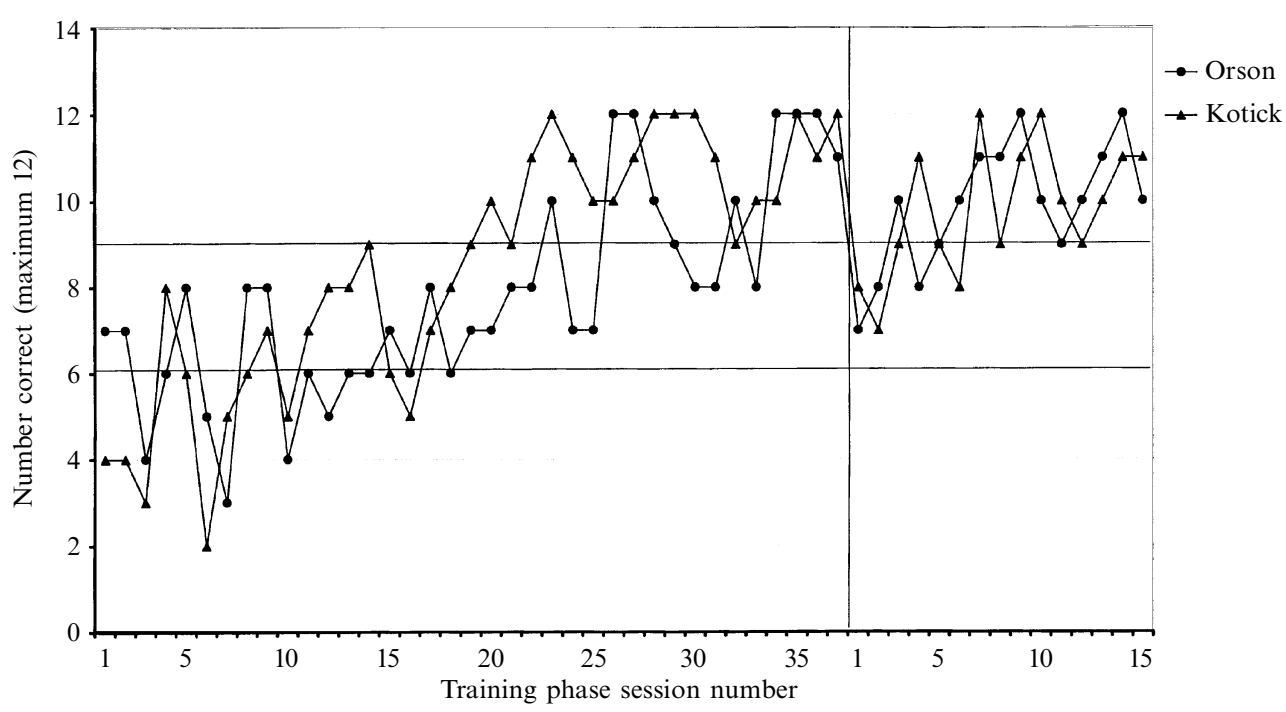

Figure 4. Performance of the sea lions during the pre-training phase and the training phase. $6 / 12$ represents chance performance and 9 out of 12 is significantly above chance. There was a brief drop in performance when the laminated pictures were replaced with computer-displayed pictures, but performance quickly returned to above-chance levels.

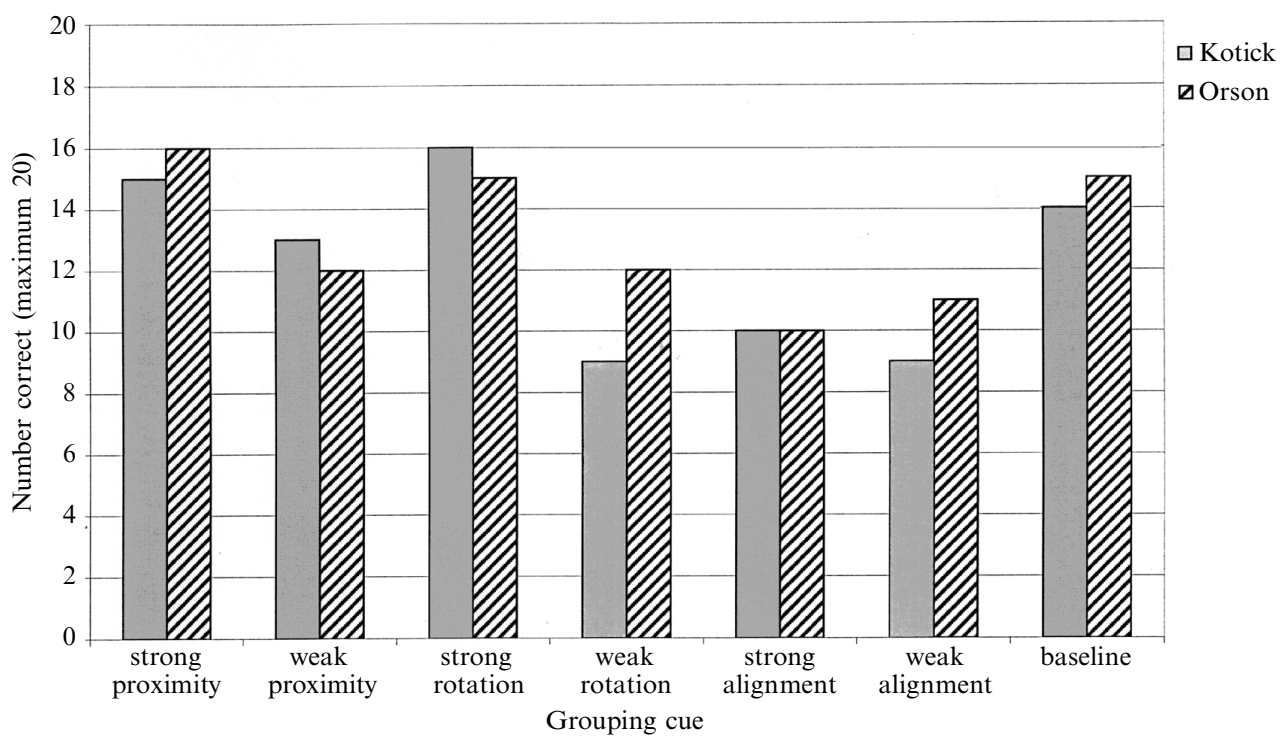

Figure 5. Performance of the sea lions on the test stimuli: 15 out of 20 represents above-chance performance.

rotation (similarity) cues, and Orson was above chance in the baseline condition. Kotick's performance approached significance in this condition $\left(\chi^{2}=3.2, p=0.074\right.$, and $p=0.115$ on a binomial test). Performance on the other stimuli was not significantly above chance. Because we were primarily interested in differences between the conditions, and despite the small number of subjects, we also conducted a 2 (cue strength) $\times 3$ (grouping principle) ANOVA on these data (excluding the baseline stimuli). This revealed a significant main effect of grouping principle $\left(F_{2,2}=52, p=0.019\right)$, but a nonsignificant main effect of cue strength $\left(F_{1,1}=16, p=0.156\right)$ and a nonsignificant interaction $\left(F_{2,2}=2.74, p=0.269\right)$. The central finding from this ANOVA is that there is 
a significant effect of grouping principle, consistent with the $\chi^{2}$ analysis, but the $F$-ratios for the other effects suggest that they may have also reached significance had we been able to run more subjects (who performed in a similar way).

\subsection{Discussion}

The results of this experiment demonstrate that the sea lions were able to generalise the horizontal/vertical discrimination to new exemplars containing real lines and to the strong versions of the proximity and similarity patterns, but their performance was at chance levels for the other patterns. This suggests that they were able to discriminate horizontal from vertical using proximity and similarity information, but that they were less sensitive to them than are human subjects. Kurylo et al (1997) draw the same conclusion about rats, with respect to proximity. It is also possible that the motivation levels of the sea lions was not particularly high and that they therefore directed their efforts at discriminations which they found easier, and so did not devote complete attention to the difficult versions of these cues. If this is true, it would be difficult to compare absolute sensitivity to the grouping information across species, but we were primarily interested whether they showed any sign of sensitivity to the cue, which, for these two cues, they plainly did.

The performance of both sea lions is at chance levels for both strengths of the alignment cue, suggesting that they do not have any sensitivity to this kind of information, at least as a cue to global orientation. The data from experiment 1 showed that human subjects find the alignment information in these patterns no less useful than the proximity or similarity information, so an explanation of the sea lions' failure on alignment cannot be attributed to these patterns providing intrinsically weaker vertical and horizontal signals. It seems that the sea lions were simply not sensitive to the alignment information provided.

The sea lions in this experiment performed in much the same way as the hooded rats used by Kurylo et al (1997), despite their greater reliance on vision, greater visual acuity, training with a range of horizontal and vertical exemplars, and attempts to rule out alternative explanations. This raises the possibility that the effect discovered by Kurylo et al is, in fact, a consequence of the grouping cue used, and not a consequence of the poor acuity of their subjects, or of the small number of training exemplars. So, of the species which have so far been tested, there is now convincing evidence of a qualitative difference in the ability to use alignment information as a global cue to orientation between humans on the one hand, and brown rats and Australian sea lions on the other. Such a distinction, of course, invites speculation about its cause.

The fact that both sea lions and rats are insensitive to global orientation defined by alignment is consistent with the possibility that only humans are sensitive to this grouping cue. If this is true, then it could be an evolved difference between humans and the other species or a consequence of the kinds of visual experiences the subjects have had. As was mentioned earlier, to be sure that such patterns of sensitivity are a consequence of species membership and not experiential factors, it would be necessary to ensure that the subjects were exposed to the same kinds of visual environments, particularly during the development of the visual system. While it is likely that the rats used by Kurylo et al developed in visually impoverished environments, this is not true of the sea lions used in the current study, who have always inhabited a rich visual world. If visual experience is the crucial difference between the humans and the non-human subjects, then it must be a particular kind of visual stimulation that humans were exposed to, but the sea lions were not, which accounts for their sensitivity to alignment information. One such possibility is text, of course, in which horizontal alignment is important (in the main language of our human subjects). We are unable to think of any other alignment information which only humans are exposed to, but the possibility 
of experienced-based differences in perceptual grouping is interesting, and suggests a range of comparisons between subjects with different kinds of visual experiences.

The other possible reason for the differences observed between humans, rats, and sea lions is that they are a consequence of visual systems adapted (by natural selection) to meet different needs. The two non-human species tested are not closely related, they have faced dramatically different selection pressures for visual sensitivities, and only one of them is strongly reliant on vision. If insensitivity to alignment information is rare among non-humans, it is surprising that it is present in both species. Understanding why only some animals have sensitivity to particular grouping cues will necessarily involve testing a wider range of species, and attempting to correlate abilities with ecological demands placed on their visual systems, and/or phylogenetic relationships. Since it is not obvious what ecological factors faced by humans, but not by rats or sea lions, may have led to them having sensitivity to alignment information, it is difficult to know which species should be compared from this perspective. Since we know that humans do have sensitivity to this cue, the most profitable approach is probably to investigate whether close relatives of humans (chimpanzees, for example) are also sensitive to alignment information. If our closest relatives are sensitive to alignment then this raises the question which other phylogenetically related species show the same sensitivity (and, indirectly, which ecological factors may have driven the evolution of such a mechanism). If chimpanzees are not sensitive to this cue, then this would dramatically increase the probability that human sensitivity to alignment is a consequence of visual experiences that only they are likely to have. In either case, it is clear from the data reported here that the mechanisms underlying perceptual grouping are dissociable.

Acknowledgements. This research was made possible only by the generous cooperation of Taronga Zoo, in particular the staff of the marine mammal division, and especially by the outstanding efforts of Andrew Irvine. Of course, we could also not have collected any data without the sea lions, so thanks also to Kotick and Orson.

\section{References}

Birch D, Jacobs G H, 1979 "Spatial contrast sensitivity in albino and pigmented rats" Vision Research $19933-937$

Bonner N, 1994 Seals and Sea Lions of the World (London: Blandford)

Gellerman L W, 1933 "Chance orders of alternating stimuli in visual discrimination experiments" Journal of Genetic Psychology 42 206-208

Ginsburg A P, 1986 "Spatial filtering and visual form perception", in Handbook of Perception and Human Performance volume II Cognitive Processes and Performance (New York: John Wiley) chapter 34

Koffka K, 1935 Principles of Gestalt Psychology (New York: Harcourt Brace)

Kurylo D D, 1997 "Time course of perceptual grouping" Perception \& Psychophysics $59142-147$

Kurylo D D, Nest J van, Knepper B, 1997 "Characteristics of perceptual grouping in rats" Journal of Comparative Psychology 111 126-134

Legg C R, 1984 "Contrast sensitivities at low spatial frequencies in the hooded rat" Vision Research $24159-161$

Livingstone M S, Hubel D H, 1987 "Psychophysical evidence for the separate channels for the perception of form, color, movement and depth" Journal of Neuroscience 73416 -3468

Schusterman R J, 1981 "Behavioral capabilities of seals and sea lions: A review of their hearing, visual, learning and diving skills" Psychological Record 31 125-143

Schusterman R J, Balliet R F, 1971 "Aerial and underwater visual acuity in the California sea lion Zalophus californianus, as a function of luminance" Annals of the New York Academy of Sciences $18837-47$

Williams P, Tarr M J, no date "RSVP: Experimental control software for MacOs [Online]", available at http://psychumbedu/rsvp/ [1998, May 4] 